\title{
Evolution of bubble size distribution, number density, and shape in semi-batch vertical gas-liquid Taylor vortex
}

\author{
Mahdi Ramezani ${ }^{1}$, Arya Haghighat ${ }^{1}$, Meesha $\operatorname{Legg}^{1}$, Dennis Vigil ${ }^{1}$, and Michael Olsen ${ }^{1}$ \\ ${ }^{1}$ Iowa State University
}

May 5, 2020

\begin{abstract}
Bubble size distribution and bubble ellipticity were measured as a function of axial position in a vertically oriented semi-batch gas-liquid Taylor vortex reactor with varying gas flow rate and inner cylinder rotation speed producing axial Reynolds numbers in the range 23.8-119 and azimuthal Reynolds numbers up to $4.2 \times 104$. The mean bubble size increases monotonically with axial distance from the bottom of the reactor at the location of gas injection. The functional form of the growth of the mean bubble size with axial position depends upon the azimuthal Reynolds number. Specifically, when the azimuthal Reynolds number is less than $1.3 \times 104$, the mean bubble size increases linearly with axial distance from the bubble injection point. In contrast, for azimuthal Reynolds numbers greater than this critical value, the mean bubble size increases with axial distance in a sigmoidal manner.
\end{abstract}

\section{Hosted file}

Ramezani et al. AICHeJ.pdf available at https://authorea.com/users/294029/articles/422191evolution-of-bubble-size-distribution-number-density-and-shape-in-semi-batch-verticalgas-liquid-taylor-vortex 